\title{
Almost Automorphic Functions on the Quantum Time Scale and Applications
}

\author{
Yongkun Li \\ Department of Mathematics, Yunnan University, Kunming, Yunnan 650091, China \\ Correspondence should be addressed to Yongkun Li; yklie@ynu.edu.cn
}

Received 28 September 2017; Accepted 23 November 2017; Published 14 December 2017

Academic Editor: Douglas R. Anderson

Copyright (c) 2017 Yongkun Li. This is an open access article distributed under the Creative Commons Attribution License, which permits unrestricted use, distribution, and reproduction in any medium, provided the original work is properly cited.

\begin{abstract}
We first propose two types of concepts of almost automorphic functions on the quantum time scale. Secondly, we study some basic properties of almost automorphic functions on the quantum time scale. Then, we introduce a transformation between functions defined on the quantum time scale and functions defined on the set of generalized integer numbers; by using this transformation we give equivalent definitions of almost automorphic functions on the quantum time scale; following the idea of the transformation, we also give a concept of almost automorphic functions on more general time scales that can unify the concepts of almost automorphic functions on almost periodic time scales and on the quantum time scale. Finally, as an application of our results, we establish the existence of almost automorphic solutions of linear and semilinear dynamic equations on the quantum time scale.
\end{abstract}

\section{Introduction}

Because the theory of quantum calculus has important applications in quantum theory (see Kac and Cheung [1]), it has received much attention. For example, since Bohner and Chieochan [2] introduced the concept of the periodicity for functions defined on the quantum time scale, quite a few authors have devoted themselves to the study of the periodicity for dynamic equations on the quantum time scale [3-6].

However, in reality, the almost periodic phenomenon is more common and complicated than the periodic one. In addition, the almost automorphy, which was introduced in the literature by Bochner in $1955[7,8]$, is a generalization of the almost periodicity and plays an important role in understanding the almost periodicity. Therefore, to study the almost automorphy of dynamic equations on the quantum time scale is more interesting and more challenging.

Recently, on almost periodic time scales or called the invariant time scales under translations, papers $[9,10]$ introduced the concept of weighted pseudo almost automorphic functions and the concept of almost automorphic functions, respectively. Several other works, for instance, papers [11-18] also studied the almost automorphy on almost periodic time scales. The almost periodic time scale is a kind of additive time scales, while the quantum time scale is not an additive time scale; it is a kind of multiplicative time scales. Therefore, the concept of almost automorphic functions on almost periodic time scales is not suitable for dealing with almost automorphic problems on the quantum time scale and all of the results obtained in [9-18] can not be directly applied to the quantum time scale's case.

Motivated by the above, our main purpose of this paper is to propose two types of definitions of almost automorphic functions on the quantum time scale, study some of their basic properties, and establish the existence of almost automorphic solutions of nonautonomous linear dynamic equations on the quantum time scale.

The organization of this paper is as follows: In Section 2, we introduce some notations and definitions of time scale calculus. In Section 3, we propose the concepts of almost automorphic functions on the quantum time scale and investigate some of their basic properties. In Section 4, we introduce a transformation and give an equivalent definition of almost automorphic functions on the quantum time scale. Moreover, following the idea of the transformation, we also give a concept of almost automorphic functions on more general time scales that can unify the concepts of almost automorphic functions on almost periodic time scales and on the quantum time scale. In Section 5, as an application of the results, we 
study the existence of almost automorphic solutions for semilinear dynamic equations on the quantum time scale. We draw a conclusion in Section 6.

\section{Preliminaries}

In this section, we shall recall some basic definitions of time scale calculus.

A time scale $\mathbb{T}$ is an arbitrary nonempty closed subset of the real numbers; the forward and backward jump operators $\sigma, \rho: \mathbb{T} \rightarrow \mathbb{T}$ and the forward graininess $\mu: \mathbb{T} \rightarrow \mathbb{R}^{+}$are defined, respectively, by

$$
\begin{aligned}
& \sigma(t):=\inf \{s \in \mathbb{T}: s>t\}, \\
& \rho(t):=\sup \{s \in \mathbb{T}: s<t\}, \\
& \mu(t)=\sigma(t)-t .
\end{aligned}
$$

A point $t$ is said to be left-dense if $t>\inf \mathbb{T}$ and $\rho(t)=t$, right-dense if $t<\sup \mathbb{T}$ and $\sigma(t)=t$, left-scattered if $\rho(t)<$ $t$, and right-scattered if $\sigma(t)>t$. If $\mathbb{T}$ has a left-scattered maximum $m$, then $\mathbb{T}^{\kappa}=\mathbb{T} \backslash m$; otherwise $\mathbb{T}^{\kappa}=\mathbb{T}$. If $\mathbb{T}$ has a right-scattered minimum $m$, then $\mathbb{T}_{\kappa}=\mathbb{T} \backslash m$; otherwise $\mathbb{T}^{k}=\mathbb{T}$.

Let $\mathbb{X}$ be a (real or complex) Banach space. A function $f$ : $\mathbb{T} \rightarrow \mathbb{X}$ is right-dense continuous or rd-continuous provided it is continuous at right-dense points in $\mathbb{T}$ and its left-sided limits exist (finite) at left-dense points in $\mathbb{T}$. If $f$ is continuous at each right-dense point and each left-dense point, then $f$ is said to be a continuous function on $\mathbb{T}$.

For $f: \mathbb{T} \rightarrow \mathbb{X}$ and $t \in \mathbb{T}^{k}, f$ is called delta differentiable at $t \in \mathbb{T}$ if there exists $c \in \mathbb{X}$ such that, for any given $\varepsilon \geq 0$, there is an open neighborhood $U$ of $t$ satisfying

$$
\|[f(\sigma(t))-f(s)]-c[\sigma(t)-s]\| \leq \varepsilon|\sigma(t)-s|
$$

for all $s \in U$. In this case, $c$ is called the delta derivative of $f$ at $t \in \mathbb{T}$ and is denoted by $c=f^{\Delta}(t)$. For $\mathbb{T}=\mathbb{R}$, we have $f^{\Delta}=$ $f^{\prime}$, the usual derivative, for $\mathbb{T}=\mathbb{Z}$ we have the backward difference operator, $f^{\Delta}(t)=\Delta f(t):=f(t+1)-f(t)$, and for $\mathbb{T}=$ $\overline{q^{\mathbb{Z}}}(q>1)$, the quantum time scale, we have the $q$-derivative:

$$
f^{\Delta}(t):=D_{q} f(t)= \begin{cases}\frac{f(q t)-f(t)}{(q-1) t}, & t \neq 0, \\ \lim _{t \rightarrow 0} \frac{f(q t)-f(t)}{(q-1) t}, & t=0 .\end{cases}
$$

Remark 1. Note that

$$
D_{q} f(0)=\frac{d f(0)}{d t}
$$

if $f$ is continuously differentiable.

A function $p: \mathbb{T} \rightarrow \mathbb{R}$ is called regressive provided $1+$ $\mu(t) p(t) \neq 0$ for all $t \in \mathbb{T}^{\kappa}$. An $n \times n$-matrix-valued function $A$ on a time scale $\mathbb{T}$ is called regressive provided $I+\mu(t) A(t)$ is invertible for all $t \in \mathbb{T}^{\mathcal{k}}$.
Definition 2 (see [19]). A time scale $\mathbb{T}$ is called an almost periodic time scale or an invariant time scale under translations if

$$
\Pi=\{\tau \in \mathbb{R}: t \pm \tau \in \mathbb{T}, \forall t \in \mathbb{T}\} \neq\{0\} .
$$

For more details about the theory of time scale calculus and the theory of quantum calculus, the reader may want to consult [1,20-22].

\section{Almost Automorphic Functions on the Quantum Time Scale}

In this section, we propose two types of concepts of almost automorphic functions on the quantum time scale and study some of their basic properties. Our first type of concepts of almost automorphic functions on the quantum time scale is as follows.

Definition 3. Let $\mathbb{X}$ be a (real or complex) Banach space and $f: \overline{q^{\mathbb{Z}}} \rightarrow \mathbb{X}$ a (strongly) continuous function. We say that $f$ is almost automorphic if, for every sequence of integer numbers $\left\{s_{n}^{\prime}\right\} \subset \mathbb{Z}$, there exists a subsequence $\left\{s_{n}\right\}$ such that

$$
g(t):=\lim _{n \rightarrow \infty} f\left(t q^{s_{n}}\right)
$$

is well defined for each $t \in \overline{q^{\mathbb{Z}}}$ and

$$
\lim _{n \rightarrow \infty} g\left(t q^{-s_{n}}\right)=f(t)
$$

for each $t \in \overline{q^{\mathbb{Z}}}$.

Remark 4. Since $\overline{q^{\mathbb{Z}}}$ has only one right-dense point 0 and all of the other points of it are isolated points, so $f: \overline{q^{\mathbb{Z}}} \rightarrow \mathbb{X}$ is a (strongly) continuous function if and only if $\lim _{t \rightarrow 0^{+}} f(t)=$ $f(0)$.

Theorem 5. If $f, f_{1}$, and $f_{2}$ are almost automorphic functions $\overline{q^{\mathbb{Z}}} \rightarrow \mathbb{X}$, then the following are true:

(i) $f_{1}+f_{2}$ is almost automorphic.

(ii) $c f$ is almost automorphic for every scalar $c$.

(iii) $f_{a}(t) \equiv f\left(t q^{a}\right)$ is almost automorphic for each fixed $a \in \mathbb{Z}$.

(iv) $\sup _{t \in \mathbb{R}}\|f(t)\|<\infty$; that is, $f$ is a bounded function.

(v) The range $R_{f}=\left\{f(t) \mid t \in \overline{q^{\mathbb{Z}}}\right\}$ of $f$ is relatively compact in $\mathbb{X}$.

Proof. The proofs of (i), (ii), and (iii) are obvious.

The proof of (iv): If (iv) is no true, then $\sup _{t \in \overline{q^{\mathbb{Z}}}}\|f(t)\|=$ $\infty$. Hence, there exists a sequence $\left\{s_{n}^{\prime}\right\} \subset \mathbb{Z}$ such that

$$
\lim _{n \rightarrow \infty}\left\|f\left(q^{s_{n}^{\prime}}\right)\right\|=\infty .
$$

Since $f$ is almost automorphic, one can extract a subsequence $\left\{s_{n}\right\} \subset\left\{s_{n}^{\prime}\right\}$ such that

$$
\lim _{n \rightarrow \infty} f\left(q^{s_{n}}\right)=\xi
$$


exists; that is, $\lim _{n \rightarrow \infty}\left\|f\left(q^{s_{n}}\right)\right\|=\|\xi\|<\infty$, which is a contradiction. The proof of (iv) is completed.

The proof of (v): For any sequence $\left\{f\left(q^{s_{n}^{\prime}}\right)\right\}$ in $R_{f}$, where $\left\{s_{n}^{\prime}\right\} \subset \mathbb{Z}$, because $f$ is almost automorphic, one can extract a subsequence $\left\{s_{n}\right\}$ of $\left\{s_{n}^{\prime}\right\}$ such that

$$
\lim _{n \rightarrow \infty} f\left(q^{s_{n}}\right)=g(1) .
$$

Thus, $R_{f}$ is relatively compact in $\mathbb{X}$. The proof is complete.

Remark 6. It is easy to see that

$$
\sup _{t \in \overline{q^{\mathbb{Z}}}}\|g(t)\| \leq \sup _{t \in \overline{q^{\mathbb{Z}}}}\|f(t)\|,
$$

and $R_{g} \subseteq \bar{R}_{f}$, where $g$ is the function that appears in Definition 3.

Theorem 7. If $f: \overline{q^{\mathbb{Z}}} \rightarrow \mathbb{X}$ is almost automorphic, define a function $f^{\star}: \overline{q^{\mathbb{Z}}} \backslash\{0\} \rightarrow \mathbb{X}$ by $f^{\star}(t) \equiv f\left(t^{-1}\right)$, if $f^{\star}(0):=$ $\lim _{n \rightarrow-\infty} f^{\star}\left(q^{n}\right)$ exists. Then $f^{\star}: \overline{q^{\mathbb{Z}}} \rightarrow \mathbb{X}$ is almost automorphic.

Proof. For any given sequence $\left\{s_{n}^{\prime}\right\} \quad \subset \mathbb{Z}$, there exists a subsequence $\left\{s_{n}\right\}$ of $\left\{s_{n}^{\prime}\right\}$ such that

$$
\lim _{n \rightarrow \infty} f\left(t q^{s_{n}}\right)=g(t)
$$

is well defined for each $t \in \overline{q^{\mathbb{Z}}}$ and

$$
\lim _{n \rightarrow \infty} g\left(t q^{-s_{n}}\right)=f(t)
$$

for each $t \in \overline{q^{\mathbb{Z}}}$.

Define a function $g^{\star}(t) \equiv g\left(t^{-1}\right), t \in \overline{q^{\mathbb{Z}}}$, and set $\sigma_{n}=$ $-s_{n}, n=1,2, \ldots$; we get

$$
\begin{aligned}
\lim _{n \rightarrow \infty} f^{\star}\left(t q^{\sigma_{n}}\right) & =\lim _{n \rightarrow \infty} f\left(t^{-1} q^{-\sigma_{n}}\right)=\lim _{n \rightarrow \infty} f\left(t^{-1} q^{s_{n}}\right) \\
& =g\left(t^{-1}\right)=g^{\star}(t), \\
\lim _{n \rightarrow \infty} g^{\star}\left(t q^{-\sigma_{n}}\right) & =\lim _{n \rightarrow \infty} g\left(t^{-1} q^{\sigma_{n}}\right)=\lim _{n \rightarrow \infty} g\left(t^{-1} q^{-s_{n}}\right) \\
& =f\left(t^{-1}\right)=f^{\star}(t)
\end{aligned}
$$

pointwise on $\overline{q^{\mathbb{Z}}}$. Since $f^{\star}(0)=\lim _{n \rightarrow-\infty} f^{\star}\left(q^{n}\right)$ exists, $f^{\star}$ : $\overline{q^{\mathbb{Z}}} \rightarrow \mathbb{X}$ is well defined and continuous. Thus, $f^{\star}(t)$ is almost automorphic. The proof is complete.

Theorem 8. Let $\mathbb{X}$ and $\mathbb{Y}$ be two Banach spaces and $f: \overline{q^{\mathbb{Z}}} \rightarrow$ $\mathbb{X}$ an almost automorphic function. If $\phi: \mathbb{X} \rightarrow \mathbb{Y}$ is a continuous function, then the composite function $\phi(f): \overline{q^{\mathbb{Z}}} \rightarrow$ $\mathbb{Y}$ is almost automorphic.

Proof. Since $f$ is almost automorphic, for any sequence $\left\{s_{n}^{\prime}\right\} \subset$ $\mathbb{Z}$, we can extract a subsequence $\left\{s_{n}\right\}$ of $\left\{s_{n}^{\prime}\right\}$ such that

$$
\lim _{n \rightarrow \infty} f\left(t q^{s_{n}}\right)=g(t)
$$

is well defined for each $t \in \overline{q^{\mathbb{Z}}}$ and

$$
\lim _{n \rightarrow \infty} g\left(t q^{-s_{n}}\right)=f(t)
$$

for each $t \in \overline{q^{\mathbb{Z}}}$.

Since $\phi(f): \overline{q^{\mathbb{Z}}} \rightarrow \mathbb{Y}$ is continuous, we have

$$
\lim _{n \rightarrow \infty} \varphi\left(f\left(t q^{s_{n}}\right)\right)=\varphi\left(\lim _{n \rightarrow \infty} f\left(t q^{s_{n}}\right)\right)=\varphi(g(t))
$$

is well defined for each $t \in \overline{q^{\mathbb{Z}}}$ and

$$
\lim _{n \rightarrow \infty} \varphi\left(g\left(t q^{-s_{n}}\right)\right)=\varphi\left(\lim _{n \rightarrow \infty} g\left(t q^{-s_{n}}\right)\right)=\varphi(f(t))
$$

for each $t \in \overline{q^{\mathbb{Z}}}$.

That is, the composite function $\phi(f): \overline{q^{\mathbb{Z}}} \rightarrow \mathbb{Y}$ is almost automorphic. The proof is complete.

Corollary 9. If $A$ is a bounded linear operator in $\mathbb{X}$ and $f$ : $\overline{q^{\mathbb{Z}}} \rightarrow \mathbb{X}$ is an almost automorphic function, then $A(f)(t)$ is also almost automorphic.

Proof. The proof is obvious.

Theorem 10. Let $f$ be almost automorphic. If $f\left(q^{n}\right)=0$ for all $n>n_{0}$ for some integer number $n_{0}$, then $f(t) \equiv 0$ for all $t \in \overline{q^{\mathbb{Z}}}$.

Proof. It suffices to prove that $f(t)=0$ for $t \leq q^{n_{0}}$. Since $f$ is almost automorphic, for the sequence of natural numbers $\mathbb{N}=\{n\}$, one can extract a subsequence $\left\{n_{k}\right\} \subset \mathbb{N}$ such that

$$
\begin{aligned}
& \lim _{k \rightarrow \infty} f\left(t q^{n_{k}}\right)=g(t), \quad \text { for each } t \in \overline{q^{\mathbb{Z}}} \backslash\{0\}, \\
& \lim _{k \rightarrow \infty} g\left(t q^{-n_{k}}\right)=f(t), \quad \text { for each } t \in \overline{q^{\mathbb{Z}}} \backslash\{0\} .
\end{aligned}
$$

It is clear that, for any $t \leq q^{n_{0}}$, we can find $\left\{n_{k j}\right\} \subset\left\{n_{k}\right\}$ with $t q^{n_{k j}}>q^{n_{0}}$ for all $j=1,2, \ldots$. Thus, $f\left(t q^{n_{k j}}\right)=0$ for all $j=$ $1,2, \ldots$. By (19), $g(t)=\lim _{j \rightarrow \infty} f\left(t q^{n_{k j}}\right)=0$ for $t \in \overline{q^{\mathbb{Z}}} \backslash\{0\}$. Hence, according to formula (20), we obtain $f(t)=0$ for $t \in$ $\overline{q^{\mathbb{Z}}} \backslash\{0\}$. Since $f$ is continuous at $t=0,0=\lim _{n \rightarrow-\infty} f\left(q^{n}\right)=$ $f(0)$. Therefore, $f(t)=0$ for $t \in \overline{q^{\mathbb{Z}}}$. The proof is complete.

Theorem 11. Let $\left\{f_{n}\right\}$ be a sequence of almost automorphic functions such that $\lim _{n \rightarrow \infty} f_{n}(t)=f(t)$ uniformly in $t \in \overline{q^{\mathbb{Z}}}$. Then $f$ is almost automorphic.

Proof. For any given sequence $\left\{s_{n}^{\prime}\right\} \subset \mathbb{Z}$, by the diagonal procedure one can extract a subsequence $\left\{s_{n}\right\}$ of $\left\{s_{n}^{\prime}\right\}$ such that

$$
\lim _{n \rightarrow \infty} f_{i}\left(t q^{s_{n}}\right)=g_{i}(t)
$$

for each $i=1,2, \ldots$ and each $t \in \overline{q^{\mathbb{Z}}}$.

We claim that the sequence of function $\left\{g_{i}(t)\right\}$ is a Cauchy sequence. In fact, for any $i, j \in \mathbb{N}$, we have

$$
\begin{aligned}
g_{i}(t)-g_{j}(t)= & g_{i}(t)-f_{i}\left(t q^{s_{n}}\right)+f_{i}\left(t q^{s_{n}}\right)-f_{j}\left(t q^{s_{n}}\right) \\
& +f_{j}\left(t q^{s_{n}}\right)-g_{j}(t),
\end{aligned}
$$


and hence

$$
\begin{aligned}
\left\|g_{i}(t)-g_{j}(t)\right\| \leq & \left\|g_{i}(t)-f_{i}\left(t q^{s_{n}}\right)\right\| \\
& +\left\|f_{i}\left(t q^{s_{n}}\right)-f_{j}\left(t q^{s_{n}}\right)\right\| \\
& +\left\|f_{j}\left(t q^{s_{n}}\right)-g_{j}(t)\right\| .
\end{aligned}
$$

For each $\varepsilon>0$, from the uniform convergence of $\left\{f_{n}\right\}$, there exists a positive integer $N(\varepsilon)$ such that, for all $i, j>N$,

$$
\left\|f_{i}\left(t q^{s_{n}}\right)-f_{j}\left(t q^{s_{n}}\right)\right\|<\varepsilon
$$

for all $t \in \overline{q^{\mathbb{Z}}}$ and all $n=1,2, \ldots$..

It follows from (21) and the completeness of the space $\mathbb{X}$ that the sequence $\left\{g_{i}(t)\right\}$ converges pointwise on $\overline{q^{\mathbb{Z}}}$ to a function, say to function $g(t)$.

Now, we will prove

$$
\begin{aligned}
& \lim _{n \rightarrow \infty} f\left(t q^{s_{n}}\right)=g(t), \\
& \lim _{n \rightarrow \infty} g\left(t q^{-s_{n}}\right)=f(t)
\end{aligned}
$$

pointwise on $\overline{q^{\mathbb{Z}}}$.

Indeed, for each $i=1,2, \ldots$, we have

$$
\begin{aligned}
\left\|f\left(t q^{s_{n}}\right)-g(t)\right\| \leq & \left\|f\left(t q^{s_{n}}\right)-f_{i}\left(t q^{s_{n}}\right)\right\| \\
& +\left\|f_{i}\left(t q^{s_{n}}\right)-g_{i}(t)\right\| \\
& +\left\|g_{i}(t)-g(t)\right\| .
\end{aligned}
$$

For any $\varepsilon>0$, we can find some positive integer $N_{0}(t, \varepsilon)$ such that

$$
\left\|f\left(t q^{s_{n}}\right)-f_{N_{0}}\left(t q^{s_{n}}\right)\right\|<\varepsilon
$$

for every $t \in \overline{q^{\mathbb{Z}}}, n=1,2, \ldots$, and $\left\|g_{N_{0}}(t)-g(t)\right\|<\varepsilon$ for

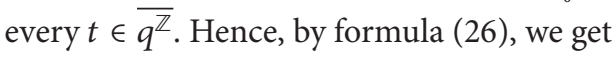

$$
\left\|f\left(t q^{s_{n}}\right)-g(t)\right\|<2 \varepsilon+\left\|f_{N_{0}}\left(t q^{s_{n}}\right)-g_{N_{0}}(t)\right\|
$$

for every $t \in \overline{q^{\mathbb{Z}}}, n=1,2, \ldots$.

In view of (21), for every $t \in \overline{q^{\mathbb{Z}}}$, there is some positive integer $M=M\left(t, N_{0}\right)$ such that

$$
\left\|f_{N_{0}}\left(t q^{s_{n}}\right)-g_{N_{0}}(t)\right\|<\varepsilon
$$

for every $n>M$. From this and (28), we obtain

$$
\left\|f\left(t q^{s_{n}}\right)-g(t)\right\|<3 \varepsilon
$$

for $n \geq N_{0}(t, \varepsilon)$.

Similarly, we can prove that

$$
\lim _{n \rightarrow \infty} g\left(t q^{s_{n}}\right)=f(t) \quad \text { for each } t \in \overline{q^{\mathbb{Z}}} .
$$

The proof is complete.
Remark 12. If we denote by $A A(\mathbb{X})$ the set of all almost automorphic functions $f: \overline{q^{\mathbb{Z}}} \rightarrow \mathbb{X}$, then by Theorem 5 , we see that $A A(\mathbb{X})$ is a vector space, and according to Theorem 11, this vector space equipped with the norm

$$
\|f\|_{A A(\mathbb{X})}=\sup _{t \in \overline{q^{\mathbb{Z}}}}\|f(t)\|
$$

is a Banach space.

Definition 13. A continuous function $f: \overline{q^{\mathbb{Z}}} \times \mathbb{X} \rightarrow \mathbb{X}$ is said to be almost automorphic in $t \in \overline{q^{\mathbb{Z}}}$ for each $x \in \mathbb{X}$, if, for each sequence of integer numbers $\left\{s_{n}^{\prime}\right\}$, there exists a subsequence $\left\{s_{n}\right\}$ such that

$$
\lim _{n \rightarrow \infty} f\left(t q^{s_{n}}, x\right)=g(t, x)
$$

exists for each $t \in \overline{q^{\mathbb{Z}}}$ and each $x \in \mathbb{X}$, and

$$
\lim _{n \rightarrow \infty} g\left(t q^{-s_{n}}, x\right)=f(t, x)
$$

exists for each $t \in \overline{q^{\mathbb{Z}}}$ and each $x \in \mathbb{X}$.

Theorem 14. If $f_{1}, f_{2}: \overline{q^{\mathbb{Z}}} \times \mathbb{X} \rightarrow \mathbb{X}$ are almost automorphic functions in $t$ for each $x \in \mathbb{X}$, then the following functions are also almost automorphic in $t$ for each $x \in \mathbb{X}$ :

(i) $f_{1}+f_{2}$

(ii) $c f_{1}: c$ is an arbitrary scalar.

Proof. The proof is obvious. We omit it here. The proof is complete.

Theorem 15. If $f(t, x)$ are almost automorphic in $t$ for each $x \in \mathbb{X}$, then

$$
\sup _{t \in \overline{q^{\mathbb{Z}}}}\|f(t, x)\|=M_{x}<\infty
$$

for each $x \in \mathbb{X}$.

Proof. Suppose the opposite. Assume, to the contrary, that

$$
\sup _{t \in \overline{q^{\mathbb{Z}}}}\left\|f\left(t, x_{0}\right)\right\|=\infty
$$

for some $x_{0} \in \mathbb{X}$. Thus, there exists a sequence of integer numbers $\left\{s_{n}^{\prime}\right\}$ such that

$$
\lim _{n \rightarrow \infty}\left\|f\left(q^{s_{n}^{\prime}}, x_{0}\right)\right\|=\infty
$$

Since $f\left(t, x_{0}\right)$ is almost automorphic in $t$, one can extract a subsequence $\left\{s_{n}\right\}$ from $\left\{s_{n}^{\prime}\right\}$ such that

$$
\sup _{t \in \overline{q^{\mathbb{Z}}}}\left\|f\left(q^{s_{n}}, x_{0}\right)\right\|=g\left(1, x_{0}\right),
$$

which is a contradiction. The proof is complete. 
Theorem 16. If $f$ is almost automorphic in $t$ for each $x \in \mathbb{X}$, then the function $g$ in Definition 13 satisfies

$$
\sup _{t \in \mathbb{R}}\|g(t, x)\|=N_{x}<\infty
$$

for each $x \in \mathbb{X}$.

Proof. The proof is obvious. We omit it here. The proof is complete.

Theorem 17. If $f$ is almost automorphic in $t$ for each $x \in \mathbb{X}$ and if $f$ satisfies the Lipschitzian condition in $x$ uniformly in $t$, that is, there exists a positive constant $L>0$ such that, for each pair $x, y \in \mathbb{X}$,

$$
\|f(t, x)-f(t, y)\|<L\|x-y\|
$$

uniformly in $t \in \overline{q^{\mathbb{Z}}}$, then $g$ satisfies the same Lipschitz condition in $x$ uniformly in $t$.

Proof. Because for each sequence of integer numbers $\left\{s_{n}^{\prime}\right\}$, there exists a subsequence $\left\{s_{n}\right\}$ such that

$$
\lim _{n \rightarrow \infty} f\left(t q^{s_{n}}, x\right)=g(t, x)
$$

exists for each $t \in \overline{q^{\mathbb{Z}}}$ and each $x \in \mathbb{X}$, for any $t \in \overline{q^{\mathbb{Z}}}$ and any given $\varepsilon>0$, we have

$$
\begin{aligned}
& \left\|g(t, x)-f\left(t q^{s_{n}}, x\right)\right\|<\frac{\varepsilon}{2}, \\
& \left\|g(t, y)-f\left(t q^{s_{n}}, y\right)\right\|<\frac{\varepsilon}{2}
\end{aligned}
$$

for $n$ sufficiently large.

Hence, for $n$ sufficiently large we find

$$
\begin{aligned}
& \|g(t, x)-g(t, y)\|=\| g(t, x)-f\left(t q^{s_{n}}, x\right) \\
& \quad+f\left(t q^{s_{n}}, x\right)-f\left(t q^{s_{n}}, y\right)+f\left(t q^{s_{n}}, y\right)-g(t, y) \| \\
& \quad<\varepsilon+L\|x-y\| .
\end{aligned}
$$

Letting $\varepsilon \rightarrow 0^{+}$, we get

$$
\|g(t, x)-g(t, y)\| \leq L\|x-y\|
$$

for each $x, y \in \mathbb{X}$. The proof is complete.

Theorem 18. Let $f: \overline{q^{\mathbb{Z}}} \times \mathbb{X} \rightarrow \mathbb{X}$ be almost automorphic in $t$ for each $x \in \mathbb{X}$ and assume that $f$ satisfies a Lipschitz condition in $x$ uniformly in $t \in \overline{q^{\mathbb{Z}}}$. Let $\varphi: \overline{q^{\mathbb{Z}}} \rightarrow \mathbb{X}$ be almost automorphic. Then the function $F: \overline{q^{\mathbb{Z}}} \rightarrow \mathbb{X}$ defined by $F(t)=$ $f(t, \varphi(t))$ is almost automorphic.

Proof. It is easy to see that, for any given sequence $\left\{s_{n}^{\prime}\right\}$, there exists a subsequence $\left\{s_{n}\right\} \subset\left\{s_{n}^{\prime}\right\}$ such that

$$
\lim _{n \rightarrow \infty} f\left(t q^{s_{n}}, x\right)=g(t, x)
$$

for each $t \in \overline{q^{\mathbb{Z}}}$ and $x \in \mathbb{X}$,

$$
\lim _{n \rightarrow \infty} \varphi\left(t q^{s_{n}}\right)=\phi(t)
$$

for each $t \in \overline{q^{\mathbb{Z}}}$,

$$
\lim _{n \rightarrow \infty} g\left(t q^{-s_{n}}, x\right)=f(t, x)
$$

for each $t \in \overline{q^{\mathbb{Z}}}$ and $x \in \mathbb{X}$, and

$$
\lim _{n \rightarrow \infty} \phi\left(t q^{-s_{n}}\right)=\varphi(t)
$$

for each $t \in \overline{q^{\mathbb{Z}}}$.

Consider the function $G: \overline{q^{\mathbb{Z}}} \rightarrow \mathbb{X}$ defined by $G(t)=$ $g(t, \phi(t)), t \in \overline{q^{\mathbb{Z}}}$. We will show that $\lim _{n \rightarrow \infty} F\left(t q^{s_{n}}\right)=G(t)$, for each $t \in \overline{q^{\mathbb{Z}}}$ and $\lim _{n \rightarrow \infty} G\left(t q^{-s_{n}}\right)=F(t)$, for each $t \in \overline{q^{\mathbb{Z}}}$.

In fact, noting that

$$
\begin{aligned}
& \left\|F\left(t q^{s_{n}}\right)-G(t)\right\|=\| f\left(t q^{s_{n}}, \varphi\left(t q^{s_{n}}\right)\right)-f\left(t q^{s_{n}}, \phi(t)\right) \\
& \quad+f\left(t q^{s_{n}}, \phi(t)\right)-g(t, \phi(t))\|\leq L\| \varphi\left(t q^{s_{n}}\right) \\
& -\phi(t)\|+\| f\left(t q^{s_{n}}, \phi(t)\right)-g(t, \phi(t)) \|,
\end{aligned}
$$

by (45) and formula (46), we get

$$
\lim _{n \rightarrow \infty} F\left(t q^{s_{n}}\right)=G(t), \quad \text { for each } t \in \overline{q^{\mathbb{Z}}} .
$$

Similarly we can prove that $\lim _{n \rightarrow \infty} G\left(t q^{-s_{n}}\right)=F(t)$ for each $t \in \overline{q^{\mathbb{Z}}}$. This completes the proof.

Before ending this section, we give the second type of concepts of almost automorphic functions on the quantum time scale as follows.

Definition 19. Let $\mathbb{X}$ be a (real or complex) Banach space and $f: \overline{q^{\mathbb{Z}}} \rightarrow \mathbb{X}$ a (strongly) continuous function. We say that $f$ is almost automorphic if, for every sequence of integer numbers $\left\{s_{n}^{\prime}\right\} \subset \mathbb{Z}$, there exists a subsequence $\left\{s_{n}\right\}$ such that

$$
g(t):=\lim _{n \rightarrow \infty} q^{s_{n}} f\left(t q^{s_{n}}\right)
$$

is well defined for each $t \in \overline{q^{\mathbb{Z}}}$ and

$$
\lim _{n \rightarrow \infty} q^{-s_{n}} g\left(t q^{-s_{n}}\right)=f(t)
$$

for each $t \in \overline{q^{\mathbb{Z}}}$.

Definition 20. A continuous function $f: \mathbb{R} \times \mathbb{X} \rightarrow \mathbb{X}$ is said to be almost automorphic in $t \in \overline{q^{\mathbb{Z}}}$ for each $x \in \mathbb{X}$, if, for each sequence of integer numbers $\left\{s_{n}^{\prime}\right\}$, there exists a subsequence $\left\{s_{n}\right\}$ such that

$$
\lim _{n \rightarrow \infty} q^{s_{n}} f\left(t q^{s_{n}}, x\right)=g(t, x)
$$

exists for each $t \in \overline{q^{\mathbb{Z}}}$ and each $x \in \mathbb{X}$, and

$$
\lim _{n \rightarrow \infty} q^{-s_{n}} g\left(t q^{-s_{n}}, x\right)=f(t, x)
$$

exists for each $t \in \overline{q^{\mathbb{Z}}}$ and each $x \in \mathbb{X}$. 
Remark 21. It is easy to check that all the results of this section hold for almost automorphic functions defined by Definitions 3 and 13 which are also valid for almost automorphic functions defined by Definitions 19 and 20.

\section{An Equivalent Definition of Almost Automorphic Functions on the Quantum Time Scale}

In this section, we will give an equivalent definition of almost automorphic functions on the quantum time scale $\overline{q^{\mathbb{Z}}}$. To this end, we introduce a notation $-\infty_{q}$ and stipulate $q^{-\infty_{q}}=0, t \pm$ $\left(-\infty_{q}\right)=t$, and $t>-\infty_{q}$ for all $t \in \mathbb{Z}$. Let $f \in C\left(\overline{q^{\mathbb{Z}}}, \mathbb{X}\right)$; we define a function $\tilde{f}: \mathbb{Z} \cup\left\{-\infty_{q}\right\} \rightarrow \mathbb{X}$ by

$$
\widetilde{f}(t)= \begin{cases}f\left(q^{t}\right), & t \in \mathbb{Z}, \\ f(0), & t=-\infty_{q} ;\end{cases}
$$

that is,

$$
f(t)= \begin{cases}\tilde{f}\left(\log _{q} t\right), & t \in q^{\mathbb{Z}} \\ \lim _{t \rightarrow 0^{+}} f(t), & t=0\end{cases}
$$

Since $f(t)$ is right continuous at $t=0$, it is clear that the above definition is well defined.

Moreover, for $f \in C\left(\overline{q^{\mathbb{Z}}} \times \mathbb{X}, \mathbb{X}\right)$, we define a function $\widetilde{f}: \mathbb{Z} \cup\left\{-\infty_{q}\right\} \times \mathbb{X} \rightarrow \mathbb{X}$ by

$$
\tilde{f}(t, x)= \begin{cases}f\left(q^{t}, x\right), & (t, x) \in \mathbb{Z} \times \mathbb{X}, \\ f(0, x), & t=-\infty_{q}, x \in \mathbb{X}\end{cases}
$$

that is,

$$
f(t, x)= \begin{cases}\tilde{f}\left(\log _{q} t, x\right), & (t, x) \in q^{\mathbb{Z}} \times \mathbb{X} \\ \lim _{t \rightarrow 0} f(t, x), & t=0, x \in \mathbb{X}\end{cases}
$$

Since $f(t, x)$ is continuous at $(0, x)$, it is clear that the above definition is well defined.

Definition 22. A function $f: \mathbb{Z} \cup\left\{-\infty_{q}\right\} \rightarrow \mathbb{X}$ is called almost automorphic if for every sequence $\left(s_{n}^{\prime}\right) \subset \mathbb{Z}$ there exists a subsequence $\left(s_{n}\right) \subset\left(s_{n}^{\prime}\right)$ such that

$$
\lim _{n \rightarrow \infty} f\left(t+s_{n}\right)=g(t)
$$

is well defined for each $t \in \mathbb{Z} \cup\left\{-\infty_{q}\right\}$, and

$$
\lim _{n \rightarrow \infty} g\left(t-s_{n}\right)=f(t)
$$

for each $t \in \mathbb{Z} \cup\left\{-\infty_{q}\right\}$.

Definition 23. A function $F:\left(\mathbb{Z} \cup\left\{-\infty_{q}\right\}\right) \times \mathbb{X} \rightarrow \mathbb{X}$ is called almost automorphic if for every sequence $\left(s_{n}^{\prime}\right) \subset \mathbb{Z}$ there exists a subsequence $\left(s_{n}\right) \subset \mathbb{Z}$ such that

$$
\lim _{n \rightarrow \infty} F\left(t+s_{n}, x\right)=G(t, x)
$$

is well defined for each $t \in \mathbb{Z} \cup\left\{-\infty_{q}\right\}$, and

$$
\lim _{n \rightarrow \infty} G\left(t-s_{n}, x\right)=F(t, x)
$$

for each $t \in \mathbb{Z} \cup\left\{-\infty_{q}\right\}$ and $x \in \mathbb{X}$.

Remark 24. We can view $\mathbb{Z} \cup\left\{-\infty_{q}\right\}$ as a kind of generalized integer number set. Obviously, the automorphic functions defined by Definitions 22 and 23 (which are defined on $\mathbb{Z} \cup$ $\left\{-\infty_{q}\right\}$ or $\left.\mathbb{Z} \cup\left\{-\infty_{q}\right\} \times \mathbb{X}\right)$ share the same properties as the ordinary automorphic functions defined on $\mathbb{Z}$ or $\mathbb{Z} \times \mathbb{X}$.

Definition 25. A function $f \in C\left(\overline{q^{\mathbb{Z}}}, \mathbb{X}\right)$ is called almost automorphic if and only if the function $\widetilde{f}(t)$ defined by (55) is almost automorphic.

Definition 26. A function $f \in C\left(\overline{q^{\mathbb{Z}}} \times \mathbb{X}, \mathbb{X}\right)$ is called almost automorphic in $t \in \overline{q^{\mathbb{Z}}}$ for each $x \in \mathbb{X}$ if and only if the function $\tilde{f}(t, x)$ defined by (57) is almost automorphic in $t \in \overline{q^{\mathbb{Z}}}$ for each $x \in \mathbb{X}$.

Obviously, Definitions 25 and 26 are equivalent to Definitions 3 and 13, respectively. Moreover, by Remark 24, all of the properties of almost automorphic functions on the quantum time scale can be directly obtained from the corresponding properties of the ordinary almost automorphic functions defined on $\mathbb{Z}$ or $\mathbb{Z} \times \mathbb{X}$.

Before ending this section, following the idea of the transformation of this section, we can propose a concept of almost automorphy on a more general time scale.

Definition 27. Let $\mathbb{T}$ be a time scale and $\tilde{\mathbb{T}}$ be an almost periodic time scale defined by Definition 2. A continuous function $f: \mathbb{T} \times \mathbb{X} \rightarrow \mathbb{X}$ is said to be almost automorphic in $t \in \mathbb{T}$ for each $x \in \mathbb{X}$, if there exists a one-to-one transformation $\varsigma: \tilde{\mathbb{T}} \rightarrow \mathbb{\mathbb { }}$ such that $\varsigma(\widetilde{\mathbb{T}})=\mathbb{T}$ and, for each sequence of integer numbers $\left\{s_{n}^{\prime}\right\} \subset \widetilde{\Pi}$, there exists a subsequence $\left\{s_{n}\right\}$ such that

$$
\lim _{n \rightarrow \infty} f\left(\varsigma\left(t+s_{n}\right), x\right)=g(\varsigma(t), x)
$$

exists for each $t \in \widetilde{\mathbb{T}}$ and each $x \in \mathbb{X}$, and

$$
\lim _{n \rightarrow \infty} g\left(\varsigma\left(t-s_{n}\right), x\right)=f(\varsigma(t), x)
$$

exists for each $t \in \widetilde{\mathbb{T}}$ and each $x \in \mathbb{X}$, where $\widetilde{\Pi}=\{\tau \in \mathbb{R}$ : $t \pm \tau \in \widetilde{\mathbb{T}}, \forall t \in \widetilde{\mathbb{T}}\}$.

Remark 28. Obviously, in Definition 27, if $\mathbb{T}$ is an almost periodic time scale defined by Definition 2 , by taking $\varsigma=$ $I$, the identity mapping, then Definition 27 coincides with Definition 3.2 in [9] and Definition 3.20 in [10], respectively, which are the definitions of almost automorphic functions on almost periodic time scales. If $T=\overline{q^{\mathbb{Z}}}$, by taking the transformation $\varsigma$ defined by (57), then Definition 27 agrees with Definition 13. Therefore, Definition 27 can unify the cases of almost periodic time scales and the quantum time scale. 


\section{Automorphic Solutions for Semilinear Dynamic Equations on the Quantum Time Scale}

In this section, we will study the existence of automorphic solutions of semilinear dynamic equations on the quantum time scale. Throughout this section, we use the letter $\mathbb{E}$ to stand for either $\mathbb{R}$ or $\mathbb{C}$.

Consider the semilinear dynamic equation on the quantum time scale:

$$
\begin{aligned}
& D_{q} x(t)=B(t) x(t)+g\left(t, x(t), x\left(t q^{-\sigma(t)}\right)\right), \\
& t \in \overline{q^{\mathbb{Z}}},
\end{aligned}
$$

where $\sigma: \mathbb{T} \rightarrow[0, \infty)_{\mathbb{T}}$ is a scalar delay function and satisfies $t-\sigma(t) \in \mathbb{T}$ for all $t \in \mathbb{T}, B(t)$ is a regressive, rd-continuous $n \times n$ matrix valued function, and $g \in C_{\mathrm{rd}}\left(\mathbb{T} \times \mathbb{E}^{2 n}, \mathbb{E}^{n}\right)$. Under transformation (57), (65) is transformed to

$$
\begin{aligned}
\Delta \widetilde{x}(n)=A(n) \tilde{x}(n)+f(n, \tilde{x}(n), \tilde{x}( & -\tau(n))), \\
n & \in \mathbb{Z} \cup\left\{-\infty_{q}\right\},
\end{aligned}
$$

and vice visa, where $A(n)=(q-1) q^{n} \widetilde{B}(n), f(n)=(q-$ 1) $q^{n} \widetilde{g}(n, \widetilde{x}(n), \widetilde{x}(n-\bar{\sigma}(n))), \tau(n)=\widetilde{\sigma}(n)$.

Clearly, $x(t)$ is a solution of (65) if and only if $\tilde{x}(n)$ is a solution of (66).

Definition 29 (see [14]). Let $A(t)$ be an $n \times n$ rd-continuous matrix value function on $\mathbb{T}$; the linear system

$$
x^{\Delta}(t)=A(t) x(t), \quad t \in \mathbb{T}
$$

is said to admit an exponential dichotomy on $\mathbb{T}$ if there exist positive constants $K_{1}, K_{2}$ and $\alpha_{1}, \alpha_{2}$ and an invertible projection $P$ commuting with $X(t)$, where $X(t)$ is principal fundamental matrix solution of (67) satisfying

$$
\begin{gathered}
\left\|X(t) P X^{-1}(s)\right\| \leq K_{1} e_{\ominus \alpha_{1}}(t, s), \\
s, t \in \mathbb{T}, t \geq s, \\
\left\|X(t)(I-P) X^{-1}(s)\right\| \leq K_{2} e_{\ominus \alpha_{2}}(s, t),
\end{gathered}
$$

$$
s, t \in \mathbb{T}, t \leq s .
$$

Theorem 30 (see [14]). Let $\mathbb{T}$ be an almost periodic time scale. Supposing that linear homogeneous system (67) admits an exponential dichotomy with the positive constants $K_{1}, K_{2}$ and $\alpha_{1}, \alpha_{2}$ and invertible projection $\mathscr{P}$ commuting with $X(t)$, where $X(t)$ is principal fundamental matrix solution of (67), then the nonhomogeneous system

$$
x^{\Delta}(t)=A(t) x(t)+f(t),
$$

has a solution $x(t)$ of the form

$$
\begin{aligned}
x(t)= & \int_{-\infty}^{t} X(t) \mathscr{P} X^{-1}(\sigma(s)) f(s) \Delta s \\
& -\int_{t}^{\infty} X(t)(1-\mathscr{P}) X^{-1}(\sigma(s)) f(s) \Delta s .
\end{aligned}
$$

Moreover, we have

$$
\|x\| \leq\left(\frac{K_{1}+\alpha_{1}}{\alpha_{1}}+\frac{K_{2}}{\alpha_{2}}\right)\|f\| .
$$

Consider the following semilinear dynamic equation on almost periodic time scale $\mathbb{T}$ :

$$
x^{\Delta}(t)=A(t) x(t)+f(t, x(t), x(t-\tau(t))),
$$

where $\tau: \mathbb{T} \rightarrow[0, \infty)_{\mathbb{T}}$ is a scalar delay function and satisfies $t-\tau(t) \in \mathbb{T}$ for all $t \in \mathbb{T}, A(t)$ is a regressive, rd-continuous $n \times n$ matrix valued function, and $f \in C_{\mathrm{rd}}\left(\mathbb{T} \times \mathbb{E}^{2 n}, \mathbb{E}^{n}\right)$. The corresponding linear homogeneous system of (72) is

$$
x^{\Delta}(t)=A(t) x(t) .
$$

We make the following assumptions:

$\left(A_{1}\right)$ Functions $\tau(t), A(t)$, and $f(t, u, v)$ are almost automorphic in $t$.

$\left(A_{2}\right)$ There exists a constant $L_{1}, L_{2}>0$ such that

$$
\begin{aligned}
& \left\|f\left(t, u_{1}, v_{2}\right)-f\left(t, u_{2}, v_{2}\right)\right\| \\
& \quad \leq L_{1}\left\|u_{1}-u_{2}\right\|+L_{2}\left\|v_{1}-v_{2}\right\|
\end{aligned}
$$

for all $t \in \mathbb{T}$ and for any vector valued functions $u$ and $v$ defined on $\mathbb{T}$.

$\left(A_{3}\right)$ Linear homogeneous system (73) admits an exponential dichotomy with the positive constants $K_{1}, K_{2}$ and $\alpha_{1}, \alpha_{2}$ and invertible projection $P$ commuting with $X(t)$, where $X(t)$ is principal fundamental matrix solution of (73).

Now, define the mapping $\Psi$ by

$$
\begin{aligned}
& (\Psi x)(t):=\int_{-\infty}^{t} X(t) \mathscr{P} X^{-1}(\sigma(s)) \\
& \cdot f(s, x(s), x(s-\tau(s))) \Delta s-\int_{t}^{\infty} X(t)(1-\mathscr{P}) \\
& \cdot X^{-1}(\sigma(s)) f(s, x(s), x(s-\tau(s))) \Delta s .
\end{aligned}
$$

The following result can be proven similar to Lemma 6 in [11]; hence we omit it.

Lemma 31. Suppose $\left(A_{1}\right)-\left(A_{3}\right)$ hold. Then the mapping $\Psi$ maps $\mathbb{A} \mathbb{A}\left(\mathbb{E}^{n}\right)$ into $\mathbb{A} \mathbb{A}\left(\mathbb{E}^{n}\right)$.

Theorem 32. Suppose $\left(A_{1}\right)-\left(A_{3}\right)$ hold. Assume further that

$$
\left(A_{4}\right)\left(\left(K_{1}+\alpha_{1}\right) / \alpha_{1}+K_{2} / \alpha_{2}\right)\left(L_{1}+L_{2}\right)<1 .
$$

Then (72) has a unique almost automorphic solution. 
Proof. For any $x, y \in \mathbb{A} \mathbb{A}\left(\mathbb{E}^{n}\right)$, we have

$$
\begin{aligned}
& \|\Psi x-\Psi y\|=\sup _{t \in \mathbb{T}} \mid \int_{-\infty}^{t} X(t) \mathscr{P} X^{-1}(\sigma(s)) \\
& \cdot[f(s, x(s), x(s-\tau(s))) \\
& -f(s, y(s), y(s-\tau(s)))] \Delta s-\int_{t}^{\infty} X(t)(I-\mathscr{P}) \\
& \cdot X^{-1}(\sigma(s))[f(s, x(s), x(s-\tau(s))) \\
& -f(s, y(s), y(s-\tau(s)))] \Delta s\left|\leq \sup _{t \in \mathbb{T}}\right| \int_{-\infty}^{t} K_{1} e_{\ominus \alpha_{1}} \\
& \cdot(t, \sigma(s))\left(L_{1}+L_{2}\right)\|x-y\| \Delta s-\int_{t}^{\infty} K_{2} e_{\ominus \alpha_{2}} \\
& \cdot(\sigma(s), t)\left(L_{1}+L_{2}\right)\|x-y\| \Delta s \mid \leq\left(\frac{K_{1}+\alpha_{1}}{\alpha_{1}}\right. \\
& \left.+\frac{K_{2}}{\alpha_{2}}\right)\left(L_{1}+L_{2}\right)\|x-y\| .
\end{aligned}
$$

Hence, $\Phi$ is a contraction. Therefore, $\Phi$ has a unique fixed point in $\mathbb{A} \mathbb{A}\left(\mathbb{E}^{n}\right)$, so (72) has a unique almost automorphic solution.

In Theorem 32, if we take $\mathbb{T}=\mathbb{Z} \cup\left\{-\infty_{q}\right\}$, then we have the following.

Theorem 33. Suppose $\left(A_{1}\right)-\left(A_{4}\right)$ hold. Then (66) has a unique almost automorphic solution, and so (65) has a unique almost automorphic solution.

Consider a linear quantum difference equation

$$
D_{q} x(t)=A(t) x(t)+f(t), \quad t \in \overline{q^{\mathbb{Z}}}
$$

where $A$ is an $n \times n$ matrix valued function and $f$ is an $n$ dimensional vector valued function. Under transformation (55), (77) transforms to

$$
\begin{aligned}
\Delta \tilde{x}(n)=(q-1) q^{n} \tilde{A}(n) \tilde{x}(n)+(q-1) & q^{n} \tilde{f}(n), \\
& n \in \mathbb{Z} \cup\left\{-\infty_{q}\right\},
\end{aligned}
$$

and vice versa.

Consider the following nonautonomous linear difference equation:

$$
x(k+1)=A(k) x(k)+f(k), \quad k \in \mathbb{Z} \cup\left\{-\infty_{q}\right\},
$$

where $A(k)$ are given nonsingular $n \times n$ matrices with elements $a_{i j}(k), 1 \leq i, j \leq n, f: \mathbb{Z} \rightarrow \mathbb{E}^{n}$ is a given $n \times 1$ vector function, and $x(k)$ is an unknown $n \times 1$ vector with components $x_{i}(k), 1 \leq i \leq n$. Its associated homogeneous equation is given by

$$
x(k+1)=A(k) x(k), \quad k \in \mathbb{Z} \cup\left\{-\infty_{q}\right\} .
$$

Similar to Definition 2.11 in [23], we give the following definition.
Definition 34. Let $U(k)$ be the principal fundamental matrix of difference system (80). System (80) is said to possess an exponential dichotomy if there exist a projection $P$, which commutes with $U(k)$, and positive constants $\eta, \nu, \alpha, \beta$ such that, for all $k, l \in \mathbb{Z} \cup\left\{-\infty_{q}\right\}$, we have

$$
\begin{aligned}
\left\|U(k) P U^{-1}(l)\right\| \leq \eta e^{-\alpha(k-l)}, \quad k \geq l, \\
\left\|U(k)(I-P) U^{-1}(l)\right\| \leq v e^{-\beta(l-k)}, \quad l \geq k .
\end{aligned}
$$

Similar to the proof of Theorem 3.1 in [12], one can easily show the following.

Theorem 35. Suppose $A(k)$ is discrete almost automorphic and a nonsingular matrix and the set $\left\{A^{-1}(k)\right\}_{k \in \mathbb{Z} \cup\left\{-\infty_{q}\right\}}$ is bounded. Also, suppose the function $f: \mathbb{Z} \cup\left\{-\infty_{q}\right\} \rightarrow \mathbb{E}^{n}$ is a discrete almost automorphic function and (80) admits an exponential dichotomy with positive constants $\nu, \eta, \beta$, and $\alpha$. Then, system (79) has an almost automorphic solution on $\mathbb{Z} \cup\left\{-\infty_{q}\right\}$.

Corollary 36. Suppose $B(n):=(q-1) q^{n} \widetilde{A}(n)+I$ is discrete almost automorphic and a nonsingular matrix and the set $\left\{B^{-1}(n)\right\}_{n \in \mathbb{Z} \cup\left\{-\infty_{q}\right\}}$ is bounded. Also, suppose the function $g:=$ $(q-1) q^{n} \tilde{f}(n): \mathbb{Z} \cup\left\{-\infty_{q}\right\} \rightarrow \mathbb{E}^{n}$ is a discrete almost automorphic function and equation

$$
\Delta y(n)=B(n) y(n)+g(n)
$$

admits an exponential dichotomy with positive constants $\nu, \eta, \beta$, and $\alpha$. Then, system (77) has an almost automorphic solution on $\overline{q^{\mathbb{Z}}}$.

\section{Conclusion}

In this paper, we proposed two types of concepts of almost automorphic functions on the quantum time scale and studied some of their basic properties. Moreover, based on the transformation between functions defined on the quantum time scale and functions defined on the set of generalized integer numbers, we gave equivalent definitions of almost automorphic functions on the quantum time scale. As an application of our results, we established the existence of almost automorphic solutions for semilinear dynamic equations on the quantum time scale. By using the methods and results of this paper, for example, one can study the almost automorphy of neural networks on the quantum time scale and population dynamical models on the quantum time scale and so on. Furthermore, by using the transformation and the set of generalized integer numbers introduced in Section 3 of this paper, or similar to Definition 27, one can propose concepts of almost periodic functions, pseudo almost periodic functions, weighted pseudo almost automorphic functions, almost periodic set-valued functions, almost periodic functions in the sense of Stepanov on the quantum time scale, and so on. 


\section{Conflicts of Interest}

The author declares that there are no conflicts of interest.

\section{Acknowledgments}

This work is supported by the National Natural Science Foundation of China under Grant 11361072.

\section{References}

[1] V. Kac and P. Cheung, Quantum Calculus, Springer, New York, NY, USA, 2002.

[2] M. Bohner and R. Chieochan, "Floquet theory for $q$-difference equations," Sarajevo Journal of Mathematics, vol. 8(21), no. 2, pp. 355-366, 2012.

[3] M. Advar and H. C. Koyuncuoglu, "Floquet theory based on new periodicity concept for hybrid systems involving $q$ difference equations," Applied Mathematics and Computation, vol. 273, pp. 1208-1233, 2016.

[4] M. Bohner and R. Chieochan, "Positive periodic solutions for higher-order functional q-difference equations," Journal of Applied Functional Analysis, vol. 8, no. 1, pp. 14-22, 2013.

[5] M. N. Islam and J. T. Neugebauer, "Existence of periodic solutions for a quantum Volterra equation," Advances in Dynamical Systems and Applications (ADSA), vol. 11, no. 1, pp. 67-80, 2016.

[6] M. Bohner and J. G. Mesquita, "Periodic averaging principle in quantum calculus," Journal of Mathematical Analysis and Applications, vol. 435, no. 2, pp. 1146-1159, 2016.

[7] S. Bochner, "Curvature and Betti numbers in real and complex vector bundles," Universitáe Politecnico de Torino, Rendiconti del Seminario Matematico, vol. 15, pp. 225-253, 1955.

[8] S. Bochner, "Continuous mappings of almost automorphic and almost periodic functions," Proceedings of the National Acadamy of Sciences of the United States of America, vol. 52, pp. 907-910, 1964.

[9] C. Wang and Y. Li, "Weighted pseudo almost automorphic functions with applications to abstract dynamic equations on time scales," Annales Polonici Mathematici, vol. 108, no. 3, pp. 225240, 2013.

[10] C. Lizama and J. G. Mesquita, "Almost automorphic solutions of dynamic equations on time scales," Journal of Functional Analysis, vol. 265, no. 10, pp. 2267-2311, 2013.

[11] C. Lizama, J. G. Mesquita, R. Ponce, and E. Toon, "Almost automorphic solutions of Volterra equations on time scales," Differential Integral Equations, vol. 30, no. (9-10), pp. 667-694, 2017.

[12] A. Milcé, "Asymptotically almost automorphic solutions for some integro-dynamic equations with nonlocal initial conditions on time scales," Dynamics of Continuous, Discrete \& Impulsive Systems. Series A. Mathematical Analysis, vol. 23, no. 1, pp. 27-46, 2016.

[13] G. M. N’Guérékata, A. Milcé, and J. C. Mado, "Asymptotically almost automorphic functions of order $n$ and applications to dynamic equations on time scales," Nonlinear Studies, vol. 23, no. 2, pp. 305-322, 2016.

[14] M. Advar, H. C. Koyuncuoglu, and Y. N. Raffoul, "Almost automorphic solutions of delayed neutral dynamic systems on hybrid domains," Applicable Analysis and Discrete Mathematics, vol. 10, no. 1, pp. 128-151, 2016.

[15] G. M. N’Guérékata, G. Mophou, and A. Milcé, "Almost automorphic mild solutions for some semilinear abstract dynamic equations on time scales," Nonlinear Studies, vol. 22, no. 3, pp. 381-395, 2015.

[16] A. Milcé and J. C. Mado, "Almost automorphic solutions of some semilinear dynamic equations on time scales," International Journal of Evolution Equations, vol. 9, no. 2, pp. 217-229, 2014.

[17] G. Mophou, G. M. N’Guérékata, and A. Milce, "Almost automorphic functions of order $\mathrm{n}$ and applications to dynamic equations on time scales," Discrete Dynamics in Nature and Society, vol. 2014, Article ID 410210, 2014.

[18] Y. Li and L. Yang, "Almost automorphic solution for neutral type high-order Hopfield neural networks with delays in leakage terms on time scales," Applied Mathematics and Computation, vol. 242, pp. 679-693, 2014.

[19] Y. Li and C. Wang, "Uniformly almost periodic functions and almost periodic solutions to dynamic equations on time scales," Abstract and Applied Analysis, vol. 2011, Article ID 341520, 2011.

[20] M. Bohner and A. Peterson, Dynamic Equations on Time Scales: An Introduction with Applications, Birkhauser, Boston, Mass, USA, 2001.

[21] M. Bohner and A. Peterson, Advances in Dynamic Equations on Time Scales, Birkhauser, Boston, Mass, USA, 2003.

[22] C. Goodrich and A. C. Peterson, Discrete Fractional Calculus, Springer-Verlag, New York, NY, USA, 2016.

[23] C. Lizama and J. G. Mesquita, "Almost automorphic solutions of non-autonomous difference equations," Journal of Mathematical Analysis and Applications, vol. 407, no. 2, pp. 339-349, 2013. 


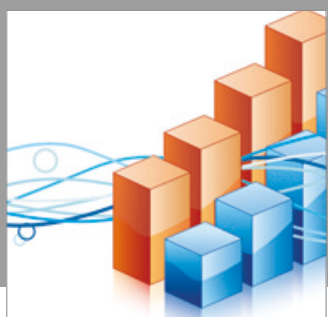

Advances in

Operations Research

vatersals

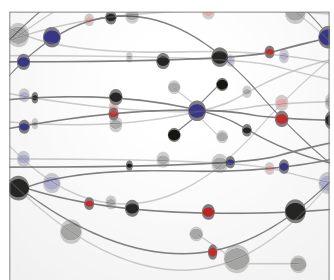

\section{The Scientific} World Journal
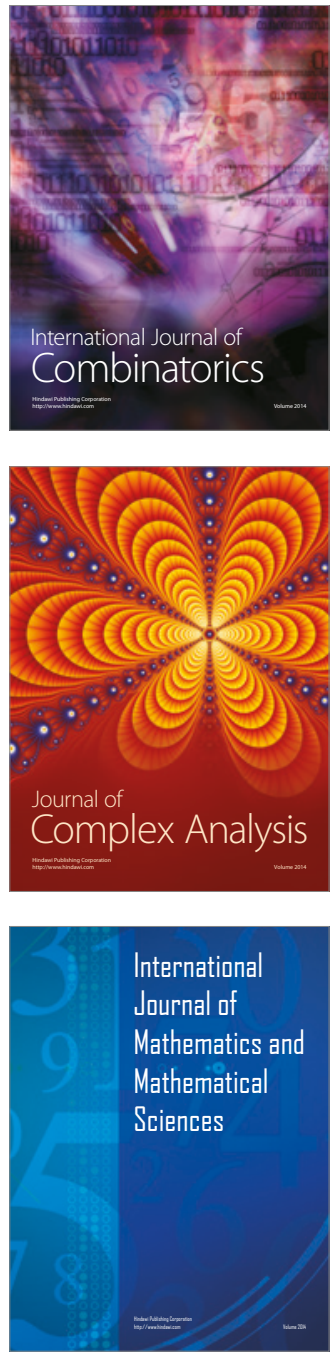
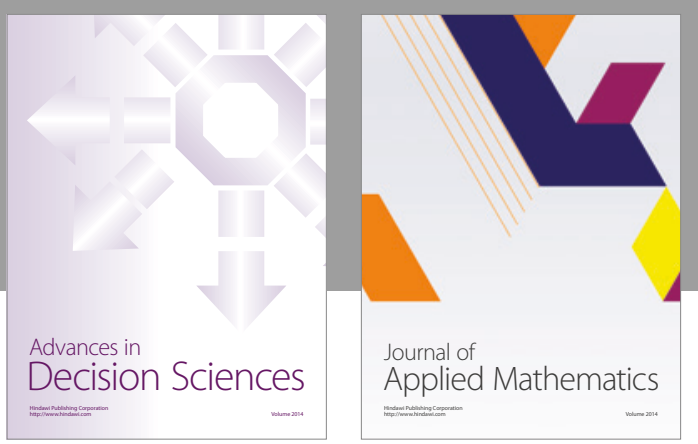

Algebra

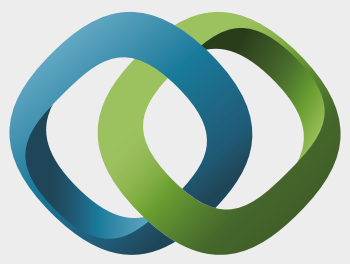

\section{Hindawi}

Submit your manuscripts at

https://www.hindawi.com
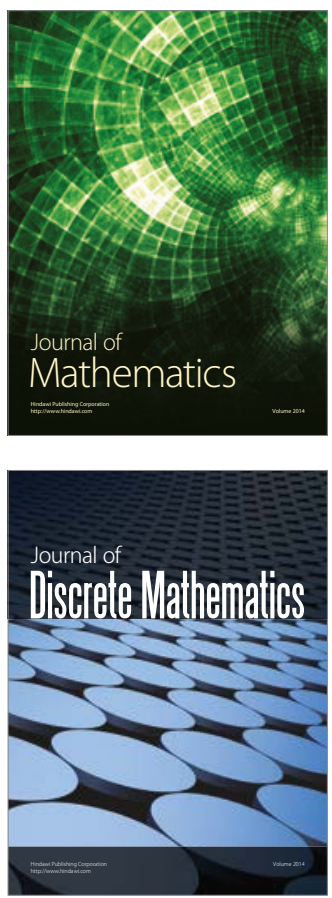

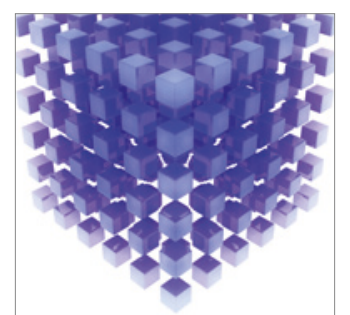

Mathematical Problems in Engineering
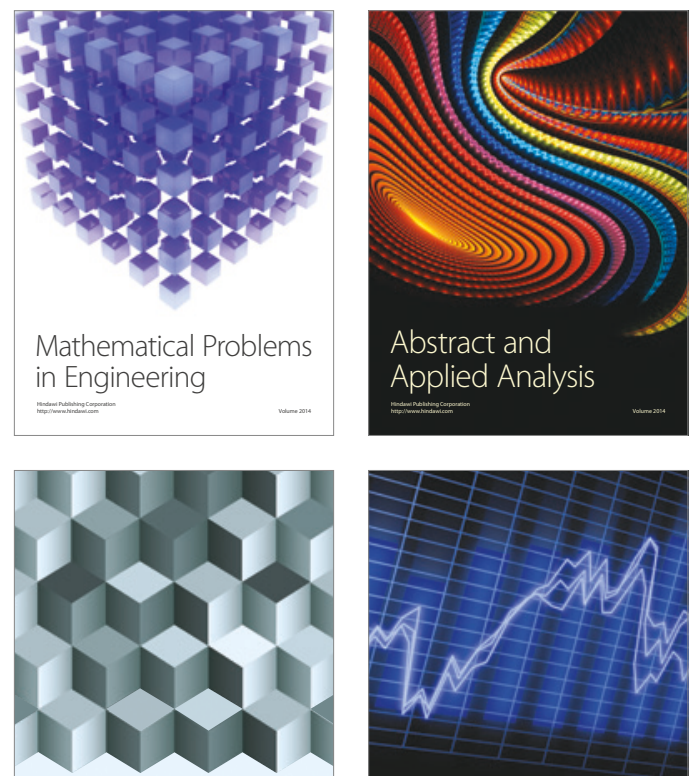

Journal of

Function Spaces

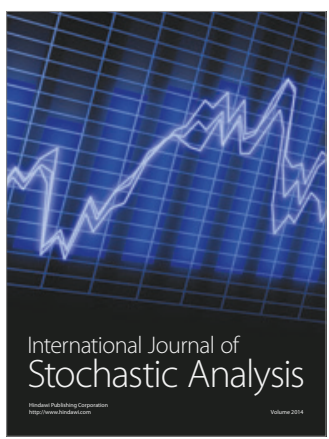

Probability and Statistics
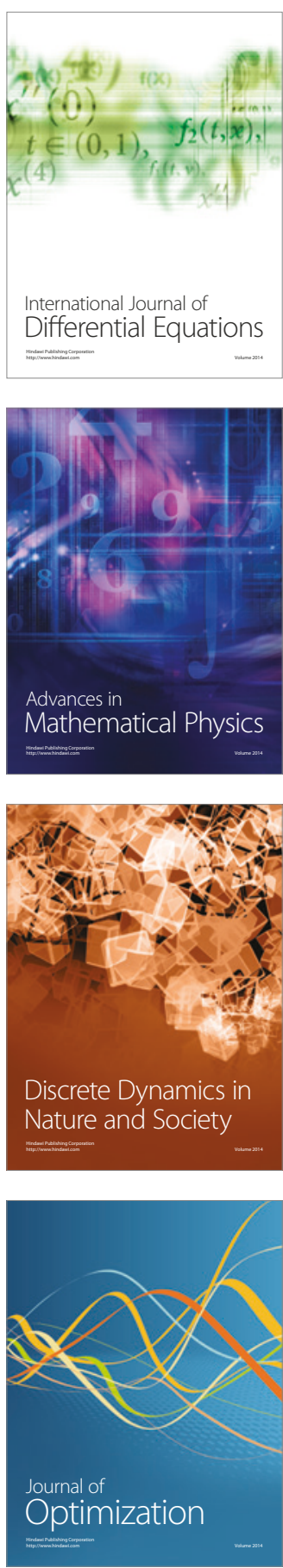